\title{
Complication sévère après injection de produit de contraste par voie intraosseuse
}

\author{
Severe Complication after Intraosseous Infusion of a Contrast Agent
}

\section{H. Thonon · D. Gusu - D. Glorieux}

Reçu le 6 janvier 2019; accepté le 30 avril 2019

(C) SFMU et Lavoisier SAS 2019

\section{Introduction}

La voie intraosseuse pour l'administration de solutions de remplissage est utilisée depuis la Seconde Guerre mondiale après le développement de la technique au début du $\mathrm{xx}^{\mathrm{e}}$ siècle. Beaucoup de publications ont remis cette technique en lumière durant la dernière décennie, particulièrement dans le domaine de l'urgence pédiatrique. Actuellement, cette technique est validée tant chez l'enfant que chez l'adulte. Cette voie d'accès est principalement utilisée chez le patient critique après l'échec des tentatives de placement de voies d'accès intravasculaires classiques. Dans certaines conditions, comme lors de la prise en charge du patient traumatisé hémodynamiquement instable, la voie d'accès intraosseuse est souvent placée sur le terrain pour permettre une perfusion rapide de solutés de remplissage ou l'administration de médicaments. Après restauration d'un équilibre hémodynamique, la question se pose de savoir si cette voie d'accès peut être utilisée pour injecter un produit de contraste avant la réalisation d'un scanner d'évaluation des lésions traumatiques. Nous décrivons ici une complication sérieuse survenue après l'usage d'un dispositif intraosseux dans ces conditions.

\section{Description du cas}

Un homme de 61 ans en état d'imprégnation éthylique était pris en charge en préhospitalier après avoir accidentellement chuté d'un toit à son domicile. Suite aux conditions de terrain et malgré une stabilité hémodynamique à l'arrivée des équipes de secours, seul un cathéter intraosseux tibial put être

\footnotetext{
H. Thonon $(\varangle)$

Service des urgences, centre hospitalier universitaire de Namur, B-5530, Namur, Belgique

e-mail : henri_thonon@hotmail.com

D. Gusu · D. Glorieux

Service des soins intensifs, grand hôpital de Charleroi (GHdC),

B-6000 Charleroi, Belgique
}

placé dans la jambe droite, dès la première tentative, pour permettre l'administration d'analgésique et d'une perfusion de remplissage. À l'arrivée à l'hôpital, le patient était nettement intoxiqué et se plaignait de douleurs diffuses. L'examen neurologique d'admission avec un score de Glasgow à 14/15 était marqué par une importante agitation associée à de la confusion, le patient mobilisait spontanément ses quatre membres, mais n'était pas collaborant. Pour préciser les lésions potentielles secondaires à la chute, un scanner du corps entier fut réalisé. La voie intraosseuse fut utilisée pour injecter $110 \mathrm{ml}$ d'iobitridol, un produit de contraste de faible osmolarité. Le produit fut administré à l'aide d'un injecteur automatique à la vitesse de $2 \mathrm{ml} / \mathrm{sec}$ et suivi d'un rinçage par $90 \mathrm{ml}$ de sérum physiologique. Le scanner révéla plusieurs fractures de vertèbres, une contusion pulmonaire, de multiples fractures costales associées à un pneumothorax. Aucun produit de contraste ne fut visualisé dans les vaisseaux sanguins après acquisition des images, et le technicien de radiologie remarqua un léger œdème au pourtour du cathéter intraosseux. Une acquisition d'images supplémentaires réalisée sur la jambe confirma l'extravasation du produit de contraste entre les muscles du mollet (Fig. 1). Dans les heures qui suivirent, un important œdème de la jambe apparut, associé à des douleurs sévères et à la disparition des pouls distaux. Quatre heures après l'injection de produit iodé, le patient dut être transféré au bloc opératoire pour une fasciotomie de décompression en urgence associée au placement d'un drain thoracique. Une grande quantité de liquide fut évacuée par le chirurgien du compartiment postérieur de la jambe, entraînant une recoloration des muscles gastrocnémiens et une réapparition des pouls distaux. Un rinçage par 31 de liquide physiologique fut également pratiqué en fin d'intervention.

\section{Discussion}

La tomodensitométrie associée à l'injection de produit de contraste est devenue un examen de routine de par le monde, 


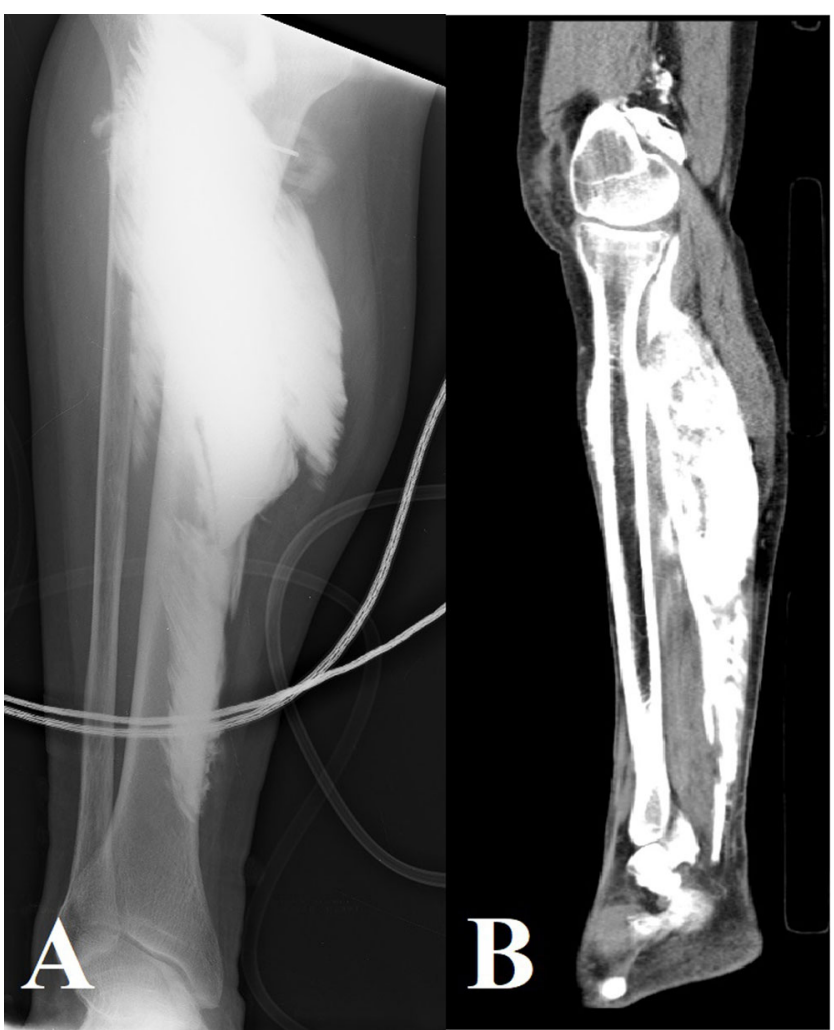

Fig. 1 Extravasation du produit de contraste entre les muscles du mollet

plus de 35 millions d'examens de ce type sont réalisés chaque année aux États-Unis. L'extravasation de produit de contraste dans les tissus sous-cutanés est une complication rarement rencontrée. Lorsque le contraste est administré par une voie veineuse périphérique, le taux de survenue est inférieur à $1 \%$ [1]. Les facteurs favorisant l'extravasation sont notamment la fragilité du réseau veineux, un nombre élevé de tentatives de mise en place du cathéter et la mobilisation du membre perfusé avant ou pendant l'injection. Pour administrer ce produit de contraste, l'American College of Radiology recommande une voie d'accès intraveineuse sûre. Dans le manuel du produit de contraste, actualisé en 2018, il est stipulé «qu'aucune grande étude ne s'est penchée sur l'utilisation de cathéter intraosseux comme voie d'injection, il n'existe dans la littérature que quelques cases reports sans complication décrite ». À notre connaissance, il existe quatre cas cliniques et une petite étude observationnelle sur le sujet [2-6]. Lors de la prise en charge du patient traumatisé, les voies d'accès vasculaires sont généralement des cathéters périphériques de gros calibre placés par les équipes de secours intervenant sur le terrain, avant l'arrivée à l'hôpital. Dans ces conditions, le taux de succès moyen de placement d'un dispositif intraveineux est approximativement de $80 \%$ [7]. Lorsqu'un cathéter intraveineux périphérique ne peut être placé, les recommandations actuelles préconisent la mise en place d'une voie intraosseuse s'il y a une nécessité d'administration rapide de soluté de remplissage ou de médicament. Les principales contre-indications à la mise en place d'un cathéter intraosseux sont une fracture osseuse, une maladie osseuse sévère, une brûlure ou une infection de la peau au niveau du site d'insertion [8]. Le principal avantage de cette voie d'accès est sa facilité et sa rapidité de placement, elle représente une alternative séduisante chez le patient instable hémodynamiquement ou lorsque l'administration des soins a lieu dans des environnements difficiles. La technique de placement est facile à apprendre et à maîtriser avec un taux de succès à la première tentative supérieur à $90 \%$ [9]. De plus, les complications après le placement d'un cathéter intraosseux sont rares, avec un taux global inférieur à $1 \%$, la principale complication étant le syndrome des loges secondaire à une extravasation du liquide injecté [8]. L'extrémité du cathéter étant située profondément dans les tissus, le mauvais placement de celle-ci à l'extérieur de la moelle osseuse passe généralement inaperçu. Même si les conséquences d'une extravasation superficielle sont généralement mineures si l'on considère l'injection de petite quantité de solution de remplissage ou d'antalgique ; la situation est différente si l'on utilise un grand volume de produit de contraste cytotoxique injecté sous pression et pouvant diffuser profondément dans les tissus comme c'est le cas lors de la réalisation d'un scanner injecté. Différents mécanismes expliquent la toxicité importante du produit de contraste extravasé : la haute osmolarité du produit injecté, la cytotoxicité directe du produit induisant une inflammation aiguë des tissus et la compression mécanique associée au volume injecté induisant un syndrome des loges. Dans la plupart des cas, l'extravasation de produit de contraste administré par une voie veineuse périphérique entraînera des symptômes immédiats et rapidement identifiables comme de la douleur et l'apparition d'un œdème local. Les lésions cutanées associées à l'extravasation peuvent aller du simple érythème à la dermohypodermite bulleuse. Cependant, même si des lésions cutanées sévères ou un syndrome des loges sont parfois observés, la plupart des extravasations de produit de contraste à partir d'un cathéter veineux périphérique sont bénignes, et un traitement conservateur est généralement suffisant. La situation est différente si le produit de contraste s'extravase à partir d'un cathéter intraosseux. L'extrémité distale de l'aiguille se trouvant davantage en profondeur, plusieurs centimètres sous la peau, les manifestations cliniques immédiates de l'extravasation sont plus discrètes. Le produit se répandant dans les loges musculaires profondes, l'apparition retardée d'un gonflement du membre et celle d'une intense douleur, caractéristiques du syndrome des loges, potentiellement plus fréquentes avec cette technique, doivent être recherchées. Même si des cases reports et des expérimentations animales décrivent l'injection de produit de contraste par voie intraosseuse sans complication 
sérieuse, le manque d'étude de grande envergure doit encourager les acteurs de la médecine d'urgence à rester prudents et à éviter l'injection par cette voie si une voie veineuse périphérique classique peut être placée, particulièrement si le dispositif intraosseux a été placé en préhospitalier. En effet, sur le terrain, les conditions de placement et les multiples mouvements du patient sur le chemin vers l'hôpital puis jusqu'à la table du scanner sont des facteurs qui augmentent le risque de déplacement du cathéter. Dans le cas décrit, l'absence de difficulté technique lors de la mise en place du cathéter et l'efficacité clinique des antalgiques administrés en préhospitalier font supposer que l'extrémité distale du dispositif a bien été placée initialement dans l'os. Le déplacement de l'aiguille dans les tissus mous s'est vraisemblablement produit lors des nombreuses mobilisations du patient jusqu'au scanner. Toutefois, un positionnement extraosseux du dispositif est possible dès sa mise en place si l'on utilise une aiguille de trop petite taille, par exemple chez un patient obèse ou à l'inverse une aiguille trop longue présentant un risque de transfixion de l'os. C'est pourquoi, après la stabilisation primaire du patient traumatisé sévère sur les lieux, le bilan secondaire à l'arrivée à l'hôpital devrait inclure une nouvelle tentative de mise en place d'un accès intraveineux périphérique ou la mise en place d'un cathéter central avant la réalisation du scanner corps entier reconnu comme la méthode de référence pour l'évaluation du patient traumatisé.

La voie intraosseuse doit donc être considérée comme un pont jusqu'au placement d'une voie d'accès dans de meilleures conditions. Évidemment, une nouvelle tentative de mise en place d'un accès vasculaire ou la réalisation d'un scanner injecté ne s'appliquent qu'au patient chez qui un équilibre hémodynamique est présent ou a pu être restauré ; dans le cas contraire, rien ne doit retarder la prise en charge chirurgicale. Dans le cas où une nouvelle voie d'accès périphérique ne peut définitivement pas être obtenue, certains auteurs proposent de confirmer le placement correct de l'extrémité du cathéter intraosseux dans la moelle en utilisant la fluoroscopie ou une acquisition de faible intensité par tomodensitométrie sur le site du cathéter avant l'injection du produit de contraste [10]. La complication sévère que nous rapportons relevant davantage de la mauvaise utilisation d'un dispositif intraosseux que d'une défaillance de celui-ci, la survenue de cette complication n'a pas entrainé de déclaration en pharmacovigilance, mais une analyse interne de la succession d'événements ayant conduit à celle-ci. Rappelons qu'en France les professionnels de santé ont l'obligation de déclarer tout effet indésirable lié à l'utilisation d'un produit de santé à l'Agence nationale de sécurité du médicament et des produits de santé (ANSM).

En conclusion, la voie d'accès intraosseuse est réellement un atout pour permettre une stabilisation du patient traumatisé sur le terrain, mais nous décrivons une complication majeure liée à l'administration de produit de contraste par cette voie. Des investigations complémentaires sont nécessaires pour valider cette pratique.

Liens d'intérêts : les auteurs déclarent ne pas avoir de liens d'intérêts.

\section{Références}

1. Wang C, Cohan R, Ellis J, et al (2007) Frequency, management, and outcome of extravasation of nonionic iodinated contrast medium in 69,657 intravenous injections. Radiology 243:80-7

2. Cambray E, Donaldson J, Shore R (1997) Intraosseous contrast infusion: efficacy and associated findings. Pediatr Radiol 27:892-3

3. Geller E, Crisci K (1999) Intraosseous infusion of iodinated contrast in an abused child. Pediatr Emerg Care 15:328-9

4. Knuth T, Paxton J, Myers D (2011) Intraosseous injection of iodinated computed tomography contrast agent in an adult blunt trauma patient. Ann Emerg Med 57:382-6

5. Ahrens K, Reeder S, Keevil J, Tupesis J (2013) Successful computed tomography angiogram through tibial intraosseous access: a case report. J Emerg Med 45:182-5

6. Winkler M, Talley C, Woodward C, et al (2017) The use of intraosseous needles for injection of contrast media for computed tomographic angiography of the thoracic aorta. J Cardiovasc Comput Tomogr 11:203-7

7. Nadler R, Gendler S, Benov A, et al (2015) Intravenous access in the prehospital settings: what can be learned from point-of-injury experience. J Trauma Acute Care Surg 79:221-6

8. Petitpas F, Guenezan J, Vendeuvre T, et al (2016) Use of intraosseous access in adults: a systematic review. Crit Care 20:102

9. Dolister M, Miller S, Borron S, et al (2013) Intraosseous vascular access is safe, effective and costs less than central venous catheters for patients in the hospital setting. J Vasc Access 14:216-24

10. Baadh A, Singh A, Choi A, et al (2016) Intraosseous vascular access in radiology: review of clinical status. Am J Roentgenol 207:241-7 